\title{
Investigation of the Effects of Plasma Treatment on the Properties of Flax Fabrics
}

\author{
Seyda CANBOLAT ${ }^{1}$, Dilek KUT ${ }^{2}$, Pinar TIRYAKI ${ }^{2}$, Filiz DEMIR ${ }^{2}$ \\ ${ }^{1}$ Istanbul Commerce University, Faculty of Engineering and Design, Department of Fashion and Textile, Istanbul, Turkey. \\ ${ }^{2}$ Uludag University, Faculty of Engineering, Department of Textile Engineering, Bursa, Turkey.
}

\begin{abstract}
In this study, flax fabrics were applied with oxygen and nitrogen plasma treatment for different execution time. After the plasma treatment, the effects of gas type and execution time on flax fabrics were investigated in terms of shear strength, hydrophility, color differences, dimensional change and surface properties. According to the results, nitrogen plasma treatment caused to increase the shear strength of flax fabrics more than oxygen plasma treatment. Furthermore, the oxygen and nitrogen plasma treatment caused to increase of hydrophility of flax fabrics and the hydrophility of samples increased with the increment in execution time of plasma treatment. The color differences results indicated that there were not significant differences in color change of flax samples. Besides, scanning electron microscope images demonstrated that the nitrogen plasma treatment provided the optimal surface modification. According to the results, plasma treatment can be improved the physical and chemical properties of flax fabrics.
\end{abstract}

Keywords: Flax, Plasma Treatment, Shear Strength, Hydrophility, Color Differences, Dimensional Change.

\section{INTRODUCTION}

The textile industry has been researching for new and alternative technologies to meet the quality, economic and eco-friendly production [1]. Plasma technology has been applied in textile industry in order to provide variety of surface modifications of textile materials. It also improves a wide range of textile properties such as hydrophobicity, dye exhaustion, and adhesion etc. [2]. Besides, plasma technology which is a clean, ecologic and dry technique is characterized by low consumption of water, energy and chemicals [3-5].

Recently, many researchers have investigated the plasma effects on textile materials. Omerogulları and Kut applied the oxygen plasma treatment on polyester fabrics in order to decrease the using of the amount of flame retardant agent. The results demonstrated that hydrophilic properties of polyester fabric were increased with the oxygen plasma treatment. Moreover, the plasma treatment allowed the reduction of the flame retardant concentration to $50 \mathrm{~g} / \mathrm{L}$ in the padding system [3].

Mossottil and et. all investigated the effect of argon and oxygen plasma treatment on wool fabrics such as anti-pilling, busting and whiteness. The results demonstrated that while the pilling of samples decreased after the plasma treatment, no significant differences between the untreated sample and plasma treated samples were found in the whiteness index [6].

Poll et.all investigated the effect on hydrophility of cotton materials treated oxygen plasma 1-100 mbar. The results showed that oxygen plasma treatment caused to increase in hydrophility of cotton materials [7].

Geyter and et.all researched the effects of a dielectric barrier discharge on polyester nonwoven fabrics in terms of pressure and execution time. The results demonstrated that the plasma condition effected the surface modification of polyester nonwovens [8].

Shahidi and et. all studied the properties (especially water repellency) of cotton was coated by a thin layer of aluminum at a low temperature plasma medium with oxygen and argon gas. The results showed that whereas argon plasma caused to increase repellent of cotton materials, oxygen plasma caused to increase hydrophilic of cotton materials [9].

In this study, oxygen and nitrogen plasma treatment carried out flax fabrics with low frequency (LF) at 0.9 mbar for 5 and 10 minutes. After the plasma treatment, the effects of gas type and execution time on flax fabrics were investigated

Corresponding Author: Şeyda CANBOLAT, Tel: +90 (212) 51141 88, E-mail: scanbolat@ticaret.edu.tr Submitted: 15 October 2014, Revised: 04 March 2015, Accepted: 01 July 2015 
in terms of shear strength, hydrophility, color differences, dimensional change and surface properties.

\section{MATERIAL AND METHOD}

\subsection{Material}

In this study, $100 \%$ flax fabric was used to research the different plasma treatment effect on the properties of fabric. The fabric weight was $245 \mathrm{~g} / \mathrm{m} 2$ and warp and weft yarn densities were $43 \mathrm{warp} / \mathrm{cm}$ and $38 \mathrm{weft} / \mathrm{cm}$, respectively. The properties of fabrics applied plasma treatment were given in Table 1.

Table 1. The properties of fabrics exposed to plasma treatment

\begin{tabular}{|c|c|}
\hline Sample code & Properties of fabric \\
\hline $\mathrm{O}_{2} 5^{\prime} \mathrm{LF}$ & $\begin{array}{l}\text { Oxygen plasma treatment at low frequency } \\
\text { for } 5 \text { minutes }\end{array}$ \\
\hline $\mathrm{O}_{2} 10^{\prime} \mathrm{LF}$ & $\begin{array}{l}\text { Oxygen plasma treatment at low frequency } \\
\text { for } 10 \text { minutes }\end{array}$ \\
\hline N 5' LF & $\begin{array}{l}\text { Nitrogen plasma treatment at low frequency } \\
\text { for } 5 \text { minutes }\end{array}$ \\
\hline $\mathrm{N} 10^{\prime} \mathrm{LF}$ & $\begin{array}{l}\text { Nitrogen plasma treatment at low frequency } \\
\text { for } 10 \text { minutes }\end{array}$ \\
\hline
\end{tabular}

\subsection{Plasma Treatment}

The plasma treatment of flax fabrics was performed with oxygen and nitrogen gas in Diener vacuum plasma. The samples were placed onto the anode with the pressure of the chamber at 0.9 mbar and the plasma treatment was performed with low frequency, at $40 \mathrm{kHz}$, for 5 and 10 minutes.

\subsection{Tear Strength Measurements}

Tear strength of samples was measured with using SDL ATLAS M008E Digital Elmandorf with ballistic pendulum method according to ASTM D11424 standard and 64000 $\mathrm{mN}$ load was attached to pendulum. The measurements of tear strength were iterated three times.

\subsection{Contact Angle Measurements}

Contact angle of samples was analyzed with optical contact angle measurement instrument such as Attension by Ksv Instrument. The velocity of digital camera was chosen as taken 80 images per second and the volume of drop was 4-6 $\mathrm{cm}^{3}$ and then the contact angles were gauged from the images. The measurements of contact angle were iterated four times.

\subsection{Contact Angle Measurements}

The color differences of flax samples were measured via using Konica Minolta Spectrophotometer CM-3600d according to Stendsby Method and and the CIELab values were calculated using illuminant D65 and 10o standard observer values.

\subsection{Dimensional Change Measurements}

The dimensional change of fabrics is observed in washing, wetting and drying process as shrinkage and elongation in the warp and weft direction of the fabrics. In the dimensional change measurements, the flax fabrics were washed with liquid detergent (Perwoll) in domestic washing machine at $30^{\circ} \mathrm{C}$ and $60^{\circ} \mathrm{C}$ according to TS 5720 EN ISO 6330 standard.

\subsection{Surface Analysis}

Physical structure of plasma treated flax fabrics was analyzed by using ZEISS/EVO 40 scanning electron microscope (SEM).

\section{RESULTS AND DISCUSSION}

\subsection{Results of Tear Strength Measurements}

The results of tear strength measurements of samples were given in Table 2.

Table 2. The results of tear strength measurements of samples

Tear Strength (N)

\begin{tabular}{lcc} 
Sample & Weft Direction & Warp Direction \\
Standard & 43.33 & 43.33 \\
$5^{\prime} \mathrm{LF} \mathrm{O}_{2}$ & 47.94 & 53.16 \\
$10^{\prime} \mathrm{LF} \mathrm{O}_{2}$ & 47.30 & 52.20 \\
$5^{\prime} \mathrm{LF} \mathrm{N}$ & 50.51 & 55.13 \\
$10^{\prime} \mathrm{LF} \mathrm{N}$ & 49.95 & 50.95 \\
\hline
\end{tabular}

The results of tear strength measurements of weft direction of all samples were lower than tear strength of warp direction of all samples. The reason of this result was considered that the warp density of fabrics was higher than the weft density 
of fabrics. The increase in number of yarn caused to increase in strength density of warp direction of all samples.

In addition, the results demonstrated that the tear strength of flax fabrics decreased with the increase in execution time of plasma treatment.

The increase in execution time of plasma treatment caused to increase the tear strength of samples. The reason of this result was deemed that the increase in plasma treatment of samples caused to damage flax fibers therefore the adhesion of fibers with each other increased.

The results were evaluated in term of the type of gas, nitrogen plasma caused more damaged than oxygen plasma according to SEM results. However the tear strength results demonstrated that commonly, the tear strength of samples treated with nitrogen gas was higher than the tear strength of samples treated with oxygen gas. The reason of this result was considered that the adhesion of fibers with each other increased with the detriment of fibers.

\subsection{The Results of Contact Angle Measurements}

The results of contact angle measurements of samples were given in Table 3.

Table 3. The results of contact angle measurements of samples

\begin{tabular}{cc}
\hline Sample & Contact Angle $\left(^{\circ}\right)$ \\
\hline Standard & 70.3 \\
$5^{\prime} \mathrm{LF} \mathrm{O}_{2}$ & 45.8 \\
$10^{\prime} \mathrm{LF} \mathrm{O}_{2}$ & 22.9 \\
$5^{\prime} \mathrm{LF} \mathrm{N}_{2}$ & 49.6 \\
$10^{\prime} \mathrm{LF} \mathrm{N}_{2}$ & 39.5 \\
\hline
\end{tabular}

The SEM results indicated that the plasma treatment caused to occur micro cracks on the surface of flax fibers and the amount of micro cracks increased with the increment in the execution of plasma treatment. It was deemed that the micro cracks caused to increase in the roughness of fibers surface. As a result of that the hydrophility of samples increased. The hydrophility of samples treated with oxygen plasma was higher than the hydrophility of samples treated nitrogen plasma, which, in turn, might result from higher damage of nitrogen gas. Besides, it was considered that the oxygen plasma treatment caused to increase the hydrophility groups such as hydroxyl, carbonyl and carboxylic acid in flax fibers.

\subsection{Results of Color Differences Measurements}

The results of color differences measurements of samples were given in Table 4.

Table 4. The results of contact angle measurements of samples

\begin{tabular}{lllll}
\hline $\begin{array}{l}\text { Sample } \\
\text { Code }\end{array}$ & $\Delta \mathbf{L}$ & $\Delta \mathbf{a}$ & $\Delta \mathbf{b}$ & $\Delta \mathbf{E}$ \\
\hline $\mathbf{5}^{\prime} \mathbf{L F} \mathbf{O}_{2}$ & 0.058 & -0.280 & 1.893 & 1.914 \\
$\mathbf{1 0}^{\prime} \mathbf{L F} \mathbf{O}_{2}$ & -0.671 & -0.219 & 1.531 & 1.686 \\
$\mathbf{5}^{\prime} \mathbf{L F} \mathbf{~ N}_{2}$ & -0.249 & -0.295 & 1.516 & 1.564 \\
$\mathbf{1 0}^{\prime} \mathbf{L F} \mathbf{~ N}_{2}$ & -0.480 & -0.323 & 2.272 & 2.345 \\
\hline
\end{tabular}

The results of spectrophotometric measurement showed that while the color differences of samples increased with the increment in execution time of nitrogen plasma treatment, the color differences of samples decreased with the increase in execution time of oxygen plasma treatment. Consequently, the value of color differences of samples increased with the plasma treatment.

\subsection{Results of Dimensional Change Measurements}

The results of dimensional change measurements of samples were given in Table 5.

Table 5. The results of dimensional change measurements of samples

Washing Temperature $\left({ }^{\circ} \mathrm{C}\right)$

\begin{tabular}{lll} 
Sample & $\mathbf{3 0}^{\circ} \mathbf{C}$ & $\mathbf{6 0}^{\circ} \mathbf{C}$ \\
Standard & $\% 4$ & $\% 4$ \\
$5^{\prime} \mathrm{LF} \mathrm{O}_{2}$ & $\% 2$ & $\% 4$ \\
$10^{\prime} \mathrm{LF} \mathrm{O}_{2}$ & $\% 4$ & $\% 4$ \\
$5^{\prime} \mathrm{LF} \mathrm{N}$ & $\% 2$ & $\% 4$ \\
$10^{\prime} \mathrm{LF} \mathrm{N}$ & $\% 2$ & $\% 4$ \\
\hline
\end{tabular}

The results demonstrated that plasma treatment generally caused to develop the shrinkage properties of flax samples. 
The reason of results was considered that the plasma treatment performed with oxygen and nitrogen gas caused to increase of the hydrophility groups such as hydroxyl, carbonyl and carboxylic acid in flax fibers, which, in turn might develop the shrinkage properties of samples.

\subsection{Results of Scanning Electron Microscope Analysis}

Figure 1 showed the results of SEM analysis of raw flax fabric and plasma treated fabrics.

The SEM images demonstrated that the damaged of flax fibers increased with the increment in the execution time of oxygen and nitrogen plasma treatment. Besides, the SEM images showed that nitrogen plasma treatment caused more damaged than oxygen plasma treatment.

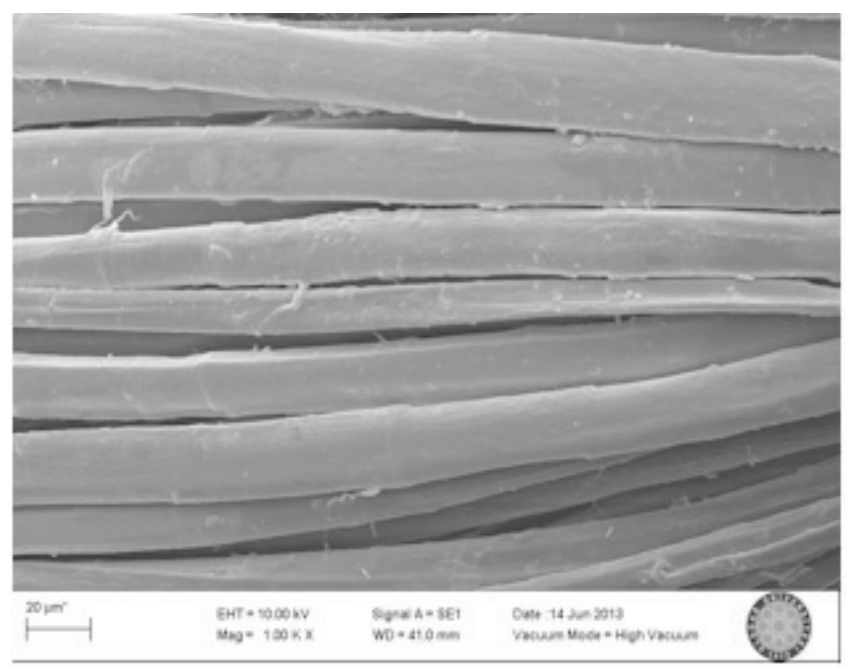

(a)

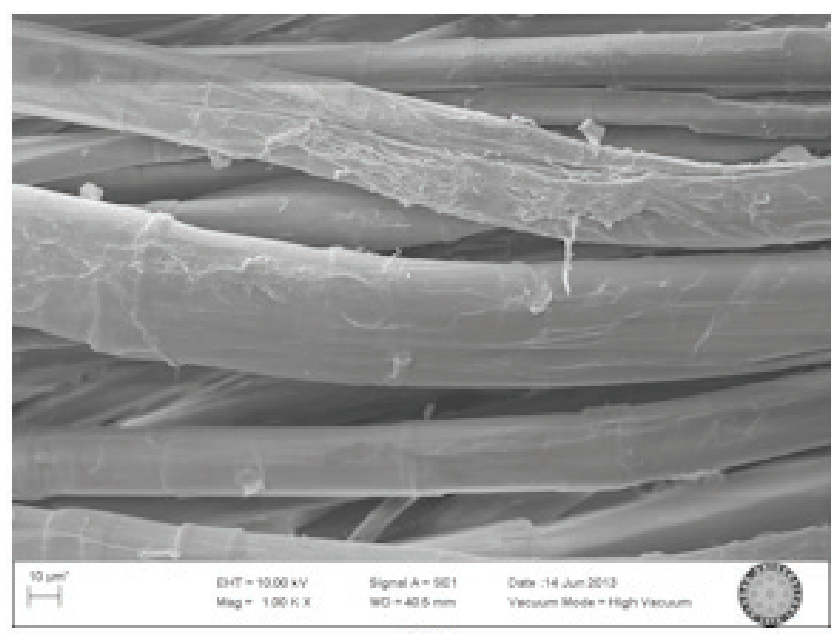

(b)

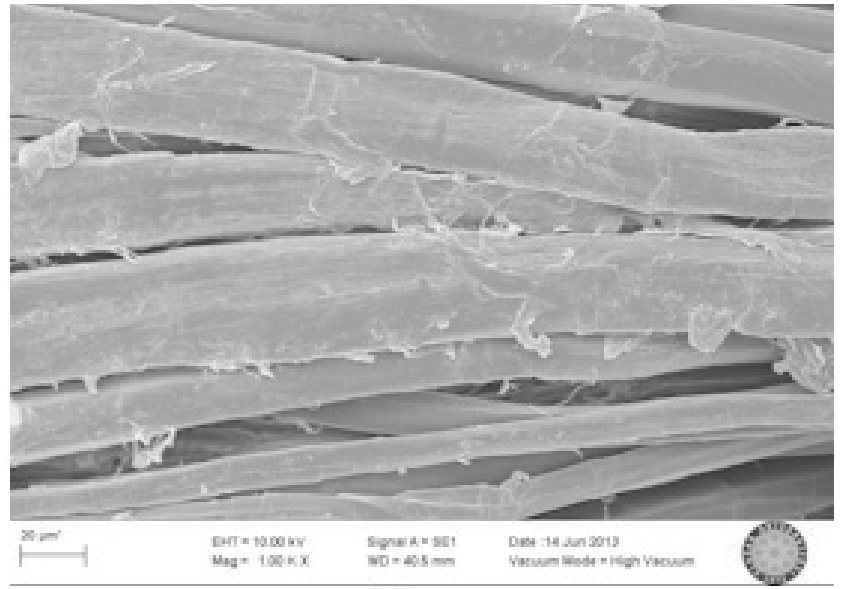

(c)

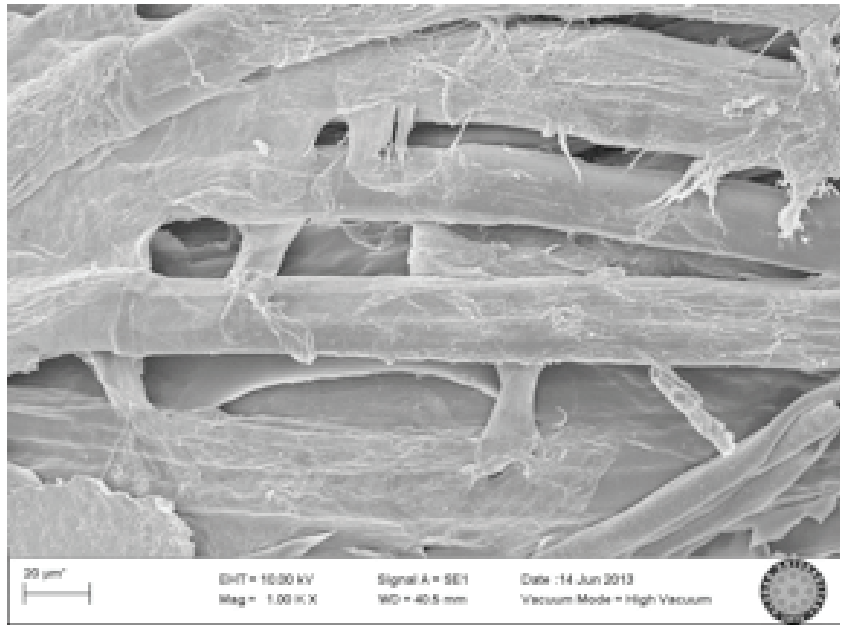

(d)

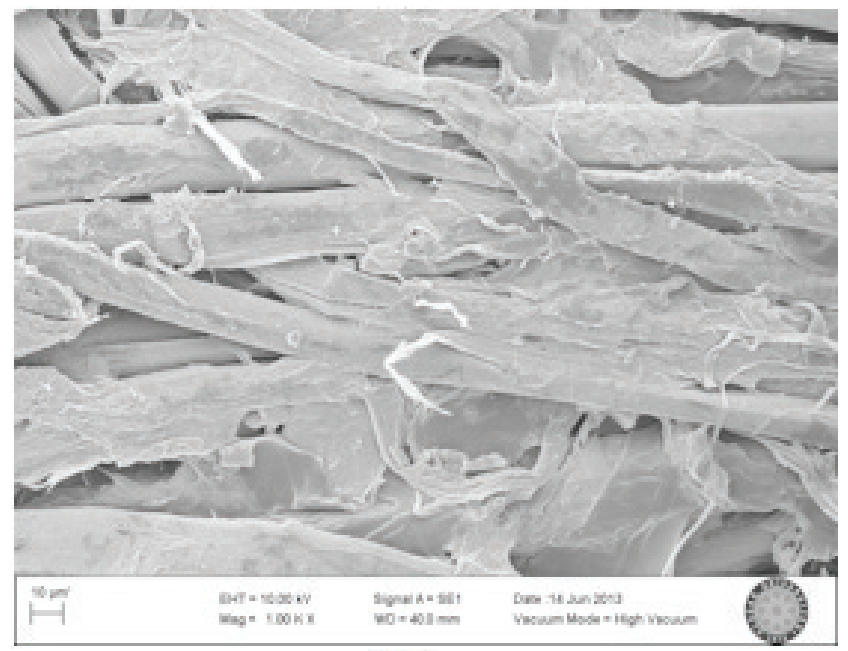

(e)

Figure 1. The SEM images of raw flax fabric (a), treated with oxygen plasma for 5 minutes (b), treated with oxygen plasma for 10 minutes (c), treated with nitrogen plasma for 5 minutes (d), treated with nitrogen plasma for 10 minutes (e) 


\section{RESULTS AND DISCUSSION}

The using of plasma treatment in textile industry has been discussed for a long time. Plasma treatment provides many advantages such as water, energy and chemical consumption and ecological production for textile industry. In this study, flax fibers were treated with oxygen and nitrogen plasma for different execution time. In order to determine the effect of plasma treatment, the shear strength, contact angle, color differences, dimensional change and surface properties of flax fabrics were investigated. The results demonstrated that nitrogen plasma treatment caused to increase the shear strength of flax fabrics more than oxygen plasma treatment. In addition, the oxygen and nitrogen plasma treatment caused to increase of hydrophility of flax fabrics and the hydrophility of samples increased with the increment in execution time of plasma treatment. The color differences results indicated that there were not significant differences in color change of flax samples. Besides, SEM images demonstrated that the nitrogen plasma treatment provided the optimal surface modification. According to the results, plasma treatment can be improved the physical and chemical properties of flax fibers.

\section{REFERENCES}

[1] Verschuren, J., Herzele, P.V., Clerck, K.D., Kiekens, P., (2005), Influence of Fiber Surface Purity on Wicking Properties of Needle-Punched Nonwoven after Oxygen Plasma Treatment, Textile Research Journal, 75(5), pp.437-441.

[2] Sahahidi, S., Rashidi, A., Ghoranneviss, M., Anvari, A., Wiener, J., (2010), Plasma Effect on Anti-Felting Properties of Wool Fabrics, Surface\&Coatings
Technology, 205 pp.349-354.

[3] Omerogulları,Z., Kut, D., (2012), Application of lowfrequency oxygen plasma treatment to polyester fabric to reduce the amount of flame retardant agent, Textile Research Journal, 82(6) pp.613-621.

[4] Bhat, N.V., Netravali, A.N., Gore, A.V., Sathianarayanan, M.P., Arolkar, G.A., Deshmukh, R.R., (2011), Surface modification of cotton fabrics using plasma technology, Textile Research Journal 81(10) pp.1014-1026.

[5] Mason,T.J, Lormier,J.P., Theory, Applications and Uses of Ultrasound in Chemistry, Ellis Horwood Limited, 1988.

[6] Mossotti,R., Lopardo, G., Innocenti,R., Mazzuchetti, G., Rombaldoni F., Montarsolo, A., (2009), Characterization of Plasma-coated Wool Fabrics, Textile Research Journal,79 (9) pp.853-861.

[7] Poll, H.U., Schladitz, U., Schreiter, S., (2001), Penetration of plasma effects into textile structures, Surface and Coatings Technology, 142 (144 ) pp.489493.

[8] Geyter, N.D., Morent, R., Leys, C., (2006), Penetration of a dielectric barrier discharge plasma into textile structures at medium pressure Belgium Plasma Sources Sci. Technol. 15 pp.78-84.

[9] Shahidi, S., Ghoranneviss, M., Moazzenchi, B., Anvari, A., Rashidi, A., (2007), Aluminum coatings on cotton fabrics with low temperature plasma of argon and oxygen, Surface \& Coatings Technology, 201 pp.5646-5650. 